\title{
Effect of Epigenetic Modifiers on Fermentation Parameters of Endophytic Fungi from Plants Growing in Uzbekistan
}

\author{
T.G. Gulyamova, L.I. Abdulmyanova, D.M. Ruzieva, G.A. Rasulova, \\ U.K. Yusupov and R.S. Sattarova* \\ Department of Biochemistry and Biotechnology of Physiologically Active Compounds, \\ Institute of Microbiology of the Academy of Sciences RU, Uzbekistan \\ *Corresponding author
}

\section{A B S T R A C T}

\section{Keywords}

Endophytic fungi, Secondary metabolites, Epi genetic modifiers, nicotinamide, Quercetin

Article Info

Accepted:

10 February 2019

Available Online:

10 March 2019
The present study aimed to investigate the effects of addition of epigenetic modifiers in the growth medium on biomass and secondary metabolites production in the suspension cultures of the endophytes Thielavia microspora MO46L with antimicrobial activity, isolated from Melissa officinalis, Penicillium concavoradulozum VE89L and Aspergillus amstelodami VR177L isolated from Vinca plants, both producing vinblastine and vincristine. The obtained data showed that quercetin and nicotinamide affected the physiological and cultural properties of studied endophytic strains as well as their metabolic profile. Results of this study also showed that quercetin can be used to induce the synthesis of vinblastine as target product in the endophytic fungi $P$. concavoradulozum VE89L and A. amstelodami VR177L.

\section{Introduction}

Endophytic fungi produce a variety of bioactive compounds of various chemical classes, including alkaloids, terpenoids, flavonoids, quinones, steroids, phenolic acids, etc., exhibiting antimicrobial, anticancer, immunomodulatory activities (Zhang et al., 2006; Nisa et al., 2015).

In particular, a plethora of different endophytes is reported to produce most of the in-demand bioactive molecules including vincristine, vinblastine, camptothecin, huperzine, podophyllotoxin, diosgenin and azadirachtin (Zhao et al., 2011b). However, the potential of endophytes as sustainable alternative production platforms is hampered due to the substantial reduction in secondary metabolite production upon repeated subculturing under axenic monoculture conditions.

As possible reasons for the attenuation lack of host stimuli in culture medium and genomic instability or silencing of the biosynthetic genes in axenic cultures have been hypothesized (Cichewicz et al., 2010). 
To enhance the yield and productivity of high value secondary metabolites from potential endophytic strains molecular (Gross et al., 2009; Henrikson et al., 2009) or cultural approaches (Hertweck, 2009) are being developed. In particular, they include optimization of cultivation parameters (composition of the medium, $\mathrm{pH}$, temperature, aeration, type of fermentation, co-cultivation, etc.) as well as adding to the cultivation medium of elicitors and epigenetic modifiers (Pettit, 2011).

Recent studies have shown that the use of epigenetic modifiers of histone deacetylase (HDAC) or DNA methyltransferase (DNMT) can lead to the expression of dormant genes changing the metabolic profile (GonzalezMenendez et al., 2016).

Earlier from plants growing in Uzbekistan a number of endophytic fungi have been isolated producing secondary metabolites with antimicrobial, cytotoxic, antioxidant and hypoglycemic activity (Abdulmyanova et al., 2015; Abdulmyanova et al., 2015; Ruzieva et al., 2017).

In the present study, the effect of addition of nicotinamide and quercetin in the growth medium on biomass and secondary metabolites production was investigated in the suspension cultures of the endophytes Thielavia microspora MO46L with antimicrobial activity, isolated from Melissa officinalis, Penicillium concavoradulozum VE89L from Vinca minor and Aspergillus amstelodami VR177L isolated from Vinca rosea, both producing vinblastine and vincristine.

\section{Materials and Methods}

The endophytic fungal strains were grown by submerged fermentation in $500 \mathrm{ml}$ flasks containing $100 \mathrm{ml}$ of Chapek-Dox liquid medium on rotary shaker at $180 \mathrm{rpm}$ and a temperature of $28{ }^{\circ} \mathrm{C}$ for 5 days. The fermentation broth of each endophyte was centrifuged at $6000 \mathrm{rpm}$ to separate the filtrate from the mycelia.

Secondary metabolites of endophytic fungi were obtained from biomass as described previously by Lang et al. (Lang et al., 2005) with modifications described by Hazalin et al (Hazalin et al., 2009).

For extraction of secondary metabolites $5 \mathrm{~g}$ of biomass of isolate was milled in a Potter homogenizer, transferred to a cone flask containing $50 \mathrm{ml}$ of ethyl acetate, and left for 24 hours on the rotary shaker at room temperature. The mixture was filtered through filter paper (Whatman \#1) and $\mathrm{Na}_{2} \mathrm{SO}_{4}(40$ $\mu \mathrm{g} / \mathrm{ml}$ ) was added. After filtration, the extract was striped to dryness on a rotary evaporator and mixed with $1 \mathrm{ml}$ of dimethyl sulfoxide (DMSO). The resulting extract was used as a stock solution and stored at $+4{ }^{\circ} \mathrm{C}$.

The metabolic profile of endophytic fungi was determined by HPLC in a $0.1 \%$ trifluoroacetic acid system with acetone on a Zorbax Eclipse XDB C-18 column (4.6 x 150 $\mathrm{mm})$ at a wavelength of $254 \mathrm{~nm}$ with a flow rate of $1 \mathrm{ml} / \mathrm{min}$. Vinblastine and vincristine preparations with the commercial name Cytoblastin and Cytocristin (India), respectively, were used as standards.

The antibacterial activity of the extracts was determined by diffusion into agar using wells. Bacillus subtilis, Pseudomonas aeruginosa, Staphylococcus aureus strains were used as test cultures. When testing extracts, a nutrient medium for bacteria was used - meat-peptone agar (MPA) (manufactured by HiMedia, India) of the following composition (per 1 liter of water): peptone $-5.00 \mathrm{~g}$., meat extract - 1.50 g., yeast extract $-1.50 \mathrm{~g}$., $\mathrm{NaCl}-5.00$ g., agar-agar -15.00 g. pH $7.4 \pm 0.2$, 
autoclaved at $1.1 \mathrm{~atm}\left(121{ }^{\circ} \mathrm{C}\right)$ for 15 minutes.

Petri dishes with MPA were seeded with a daily suspension of the test culture in physiological solution with a concentration of $1 \times 10^{6}$ cells, dried and cut out agar disks (5 $\mathrm{mm}$ diameter) and placed in $300 \mu \mathrm{l}$ of the extract in triplicate. Then the cups were incubated at $37{ }^{\circ} \mathrm{C}$ for 24-48 hours. Gentamicin sulfate at a concentration of 10 $\mu \mathrm{g} / \mathrm{ml}$ served as a positive control. The diameters of zones of inhibition were measured in millimeters.

\section{Results and Discussion}

A number of reports have described various effects of epigenetic low-molecular-weight modifiers of DNA methyltransferase (DNMT) and histone deacetylase (HDAC, sirtuins) activity on the endophytic fungi final growth profile including an increase in growth titers, chemical diversity and/or induction of new compounds (Gonzalez-Menendez et al., 2016; Vervoort et al., 2011; Chen et al., 2013; Asai et al., 2015). To study the effect on biomass and secondary metabolites production we have used nicotinamide as an epigenetic modifier, noncompetitively inhibiting the activity of sirtuins, and quercetin, a natural compound of flavonoid nature, acting as an activator of sirtuins.

After 5 days of exogenous addition of modifiers in the growth medium to a final concentration of $100 \mu \mathrm{M}$, clear and significant changes were found in the final morphology and titer of biomass, the content of metabolites and their diversity in $P$. concavoradulozum VE89L, T. microspora MO46L and A. amstelodami VR177L. As can be seen from the data presented in Figure 1-3, in the presence of nicotinamide the $P$. concavoradulozum VE89L and T. microspora MO46L acquire bright maroon and dark green pigmentation, respectively. It should be noted that this pigmentation was typical for them initially, but after several generations they lost the ability to form pigments. When adding quercetin, brown pigmentation appears in all three cultures.

The macro- and micromorphological nature of growth in the presence of quercetin and nicotinamide in endophytic strains also varied in density of suspension culture, namely in the size of macrocolonies and in the structure of mycelium.

In the mycelium of the studied endophytic fungi, quercetin causes an increase in the content of intracellular inclusions what is accompanied by thickening of the hyphae, while addition of nicotinamide contributes to the branching and lengthening of the hyphae of the mycelium (Fig. 4).

As can be seen from the data presented in Table 1, with the addition of modifiers, there are changes in the titers of biomass and secondary metabolites in all three strains.

Thus, addition of quercetin causes a significant increase of biomass in cultures of T. microspora MO46L and $P$. concavoradulozum VE89L but does not affect the biomass of $A$. amstelodami VR177L, however the level of secondary metabolites increases markedly in all three strains.

The addition of nicotinamide is also accompanied by a noticeable increase in the biomass titer in the same strains, but the level of secondary metabolites almost does not change.

Thus, nicotinamide and quercetin affect the growth of biomass and secondary metabolites production in different ways. Induction of biomass growth with quercetin is accompanied by a corresponding increase in 
the content of secondary metabolites in two strains - T. microspora MO46L and $P$. concavoradulozum VE89L while nicotinamide induced mainly biomass growth. In the case of A. amstelodami VR177L both modifiers did not affect biomass but contributed to increase of production of secondary metabolites.

It should be mentioned that similar effects were noted earlier in several reports. So, when screening 13 endophytic strains of fungi isolated from Dothideaceae species, the addition of 14 epigenetic modifiers, including nicotinamide and quercetin, has led to significant changes in the final growth morphology, changes in product titer and the formation of new peaks of metabolites in 3 strains after double treatment with modifiers (Gonzalez-Menendez et al., 2016). It was reported that nicotinamide induced production of secondary metabolites chaetophenol $\mathrm{G}$ and cancrolides A and B in endophytic fungus Chaetomium cancroideum (Asai et al., 2015).

As mentioned above, $P$. concavoradulozum VE89L and $A$. amstelodami VR177L initially were selected as potential producers of cytotoxic compounds. Moreover, it was established that supposable cytotoxic activity appears to be due to vincristine and vinblastine that they produce in trace amounts (Abdulmyanova et al., 2015).

As can be seen from data on HPLC profile of the extracts from $P$. concavoradulozum VE89L and A. amstelodami VR177L (Fig. 5, $6)$, there are total changes in the number and intensity of peaks in the presence of both modifiers, but addition of quercetin to the growth medium accompanied by a sharp increasing of the intensity of the peaks corresponding to vinblastine in both strains and decreasing vincristine peak in $P$. concavoradulozum VE89L.
It should be noted that similar data was obtained from the study of the effect of modifiers on the metabolic profile of Aspergillus fumigatus (GA-L7) isolated from Grewia asiatica L. It was established that addition of valproic acid as epigenetic modificator resulted by 10 folds increase in the production of fumiquinazoline $\mathrm{C}$ synthesizing in the original culture in trace amounts. It turned out that during the processing of a culture with this modifier 5 genes involved in the biosynthesis of fumiquinazoline $\mathrm{C}$ are overexpressed (Magotra et al., 2017).

Also, it was previously shown that the addition of 5-azacytidine and suberolanilide modifiers to the culture medium of endophytic fungi from Datura stramonium L., producing tropane alkaloids, induces the production of mycotoxins in the endophytic strain Alternaria sp. (Sun et al., 2012).

In our previous studies it was observed that endophytic strain $T$. microspora MO46L isolated from leaf of Melissa officinalis has a rather high activity against Staphylococcus aureus. In present study the effect of modifiers on the production of antibacterial secondary metabolites was investigated. It was appeared that although quercetin and nicotinamide affect biomass and secondary metabolites titers as well as diversity of metabolites, produced by $T$. microspora MO46L, antibacterial activity of extracts obtained from $T$. microspora MO46L cultures, grown in the presence of modifiers, remains at the control level (Fig. 7).

Thus, obtained data indicate that quercetin and nicotinamide affect the physiological and cultural properties of studied endophytic strains as well as their metabolic profile.

Results of this study also show that quercetin can be used to induce the synthesis of 
vinblastine as target product in the endophytic fungi $P$. concavoradulozum VE89L and $A$. amstelodami VR177L isolated from Vinca plants widely known as natural resources of vinca alkaloids.

Table.1 The effect of modifiers addition on the titers of biomass and secondary metabolites production in endophytic fungi T. microspore MO46L, P. concavoradulozumVE89L, and A. amstelodamiVR177L

\begin{tabular}{|l|c|c|c|}
\hline Endophyte strain & Control & Quercetin & Nicotinamide \\
\hline & \multicolumn{2}{|c|}{ T. microspora MO46L } \\
\hline Biomass rawweight, $\mathbf{g}$ & 1,6 & 4,1 & 2,2 \\
\hline Metabolites dw, mg & 0,007 & 0,094 & 0,015 \\
\hline \% extract /g biomass & 0,44 & 2,29 & 0,68 \\
\hline & P. concavoradulozum VE89L & 4,2 \\
\hline Biomass rawweight, g & 1,5 & 8,39 & 0,010 \\
\hline Metabolites dw,mg & 0,004 & 0,110 & 0,24 \\
\hline \% extract /g biomass & 0,27 & 1,31 & 4,3 \\
\hline & $\mathbf{A . ~ a m s t e l o d a m i V R 1 7 7 L}$ & 0,056 \\
\hline Biomass rawweight, g & 4,5 & 4,5 & 1,3 \\
\hline Metabolites dw,mg & 0,041 & 0,073 & \\
\hline \% extract /g biomass & 0,91 & 1,6 & \\
\hline
\end{tabular}

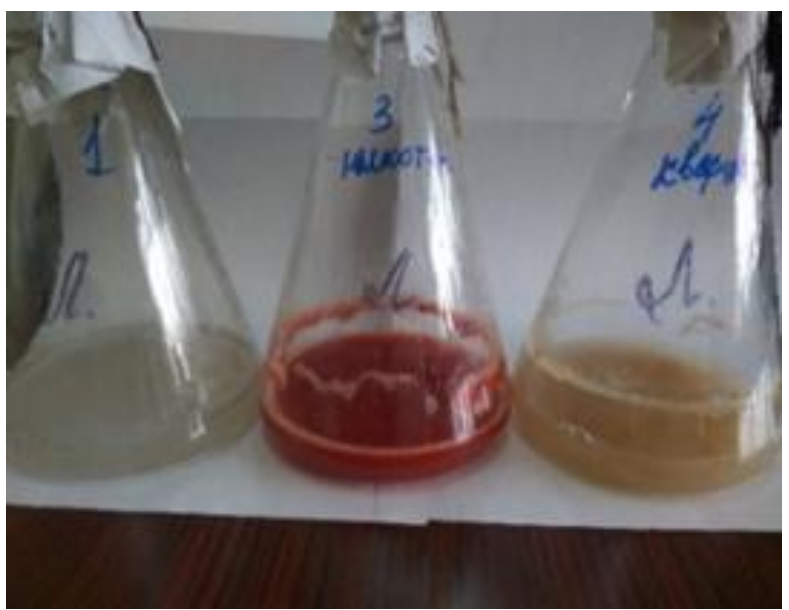

(a) (b)

(c)

Figure.1 Fermentation flasks obtained at cultivation of P. concavoradulozum VE89L in the presence of (a) - control, (b) - nicotinamide, (c) - quercetin 


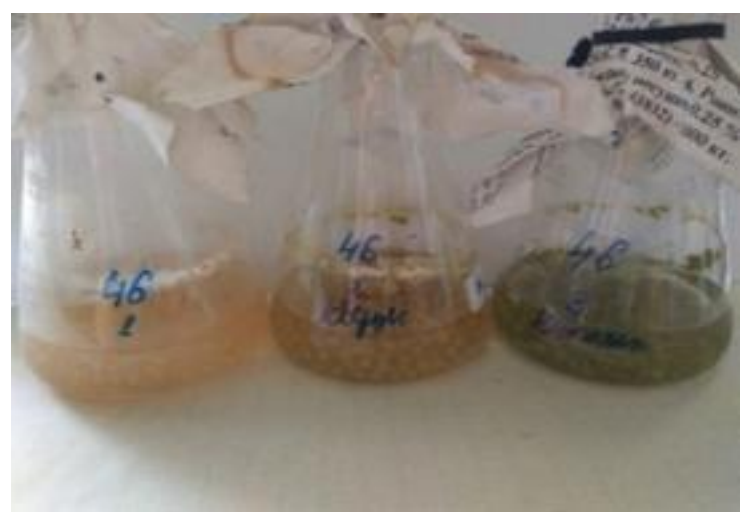
(a)
(b)
(c)

Figure.2 Fermentation flasks obtained at cultivation of T. microspora MO46L in the presence of (a) - control, (b) - quercetin, (c) - nicotinamide

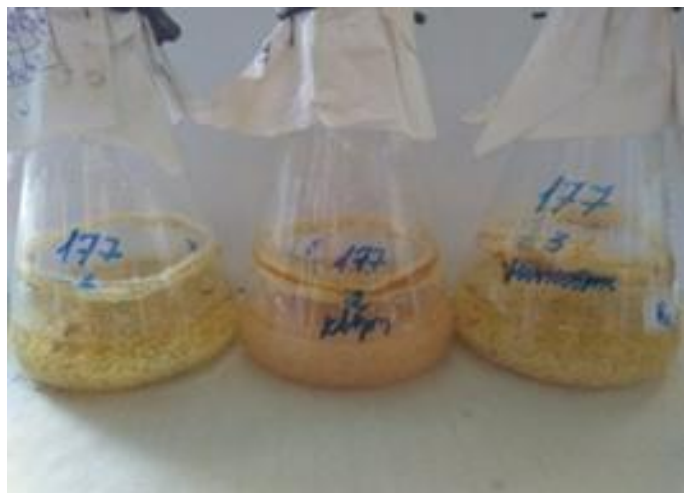

(a) (b)

Figure.3 Fermentation flasks obtained at cultivation of $A$. amstelodamiVR177L in the presence of (a) - control, (b) - quercetin, (c) - nicotinamide

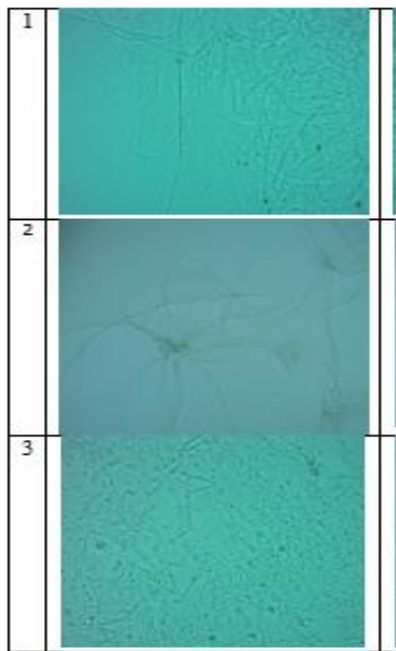

(a)

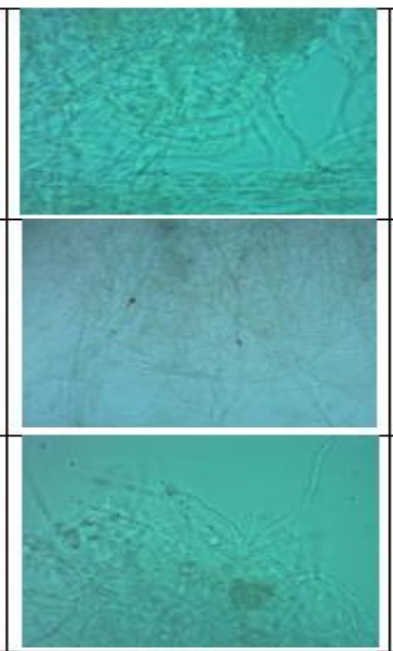

(b)

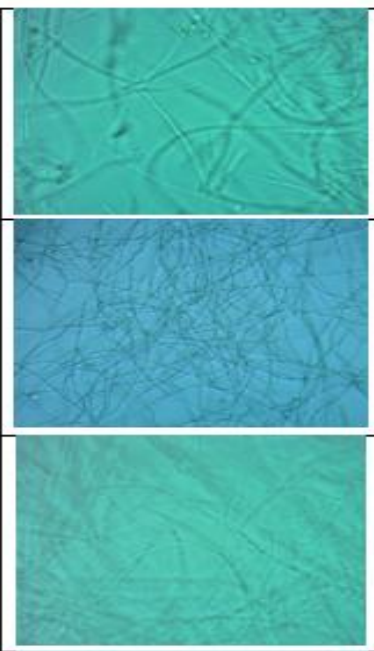

(c)

Figure.4 Morphology of mycelium obtained at cultivation of T. microsporaMO46L (1), $P$. concavoradulozum VE89L (2), and A. amstelodamiVR177L (3) in the presence (a) - control, (b) - quercetin, (c) - nicotinamide (SW 16x40) 


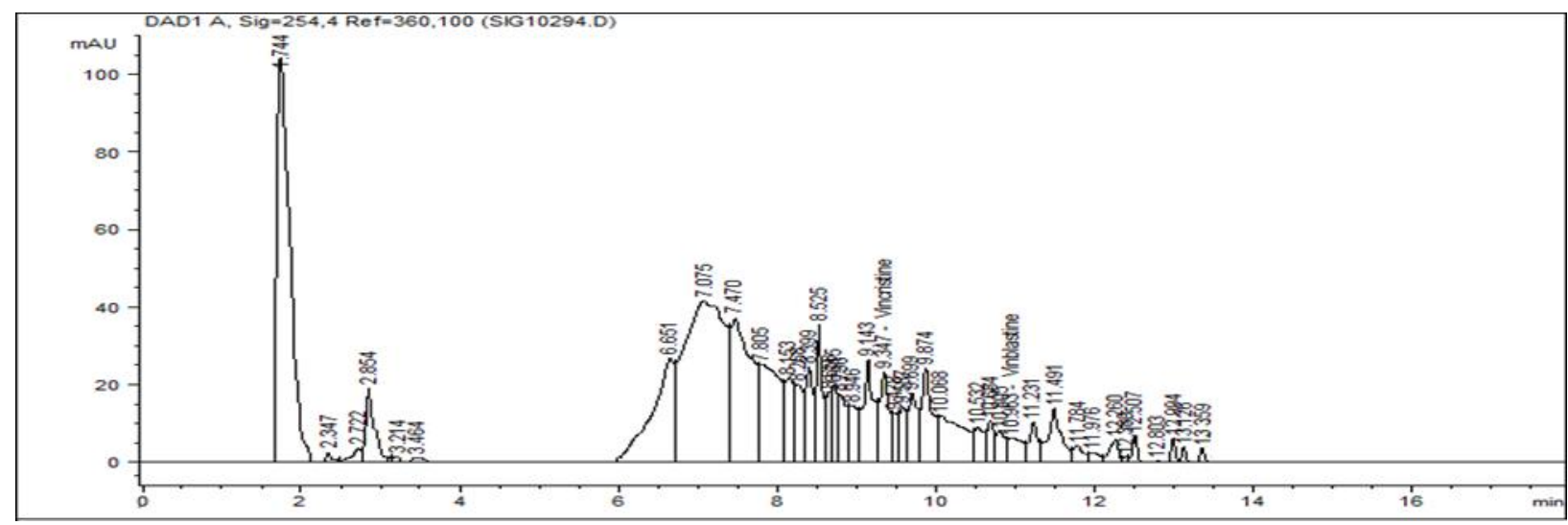

(a)

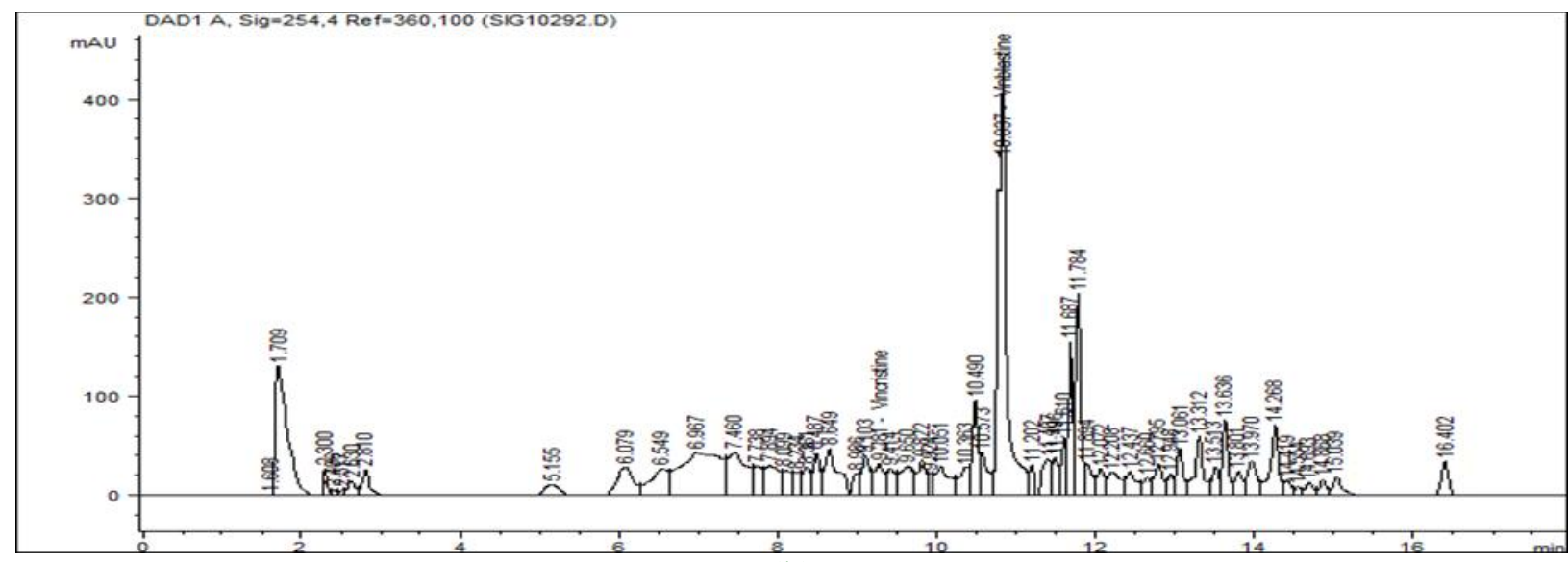

(b)

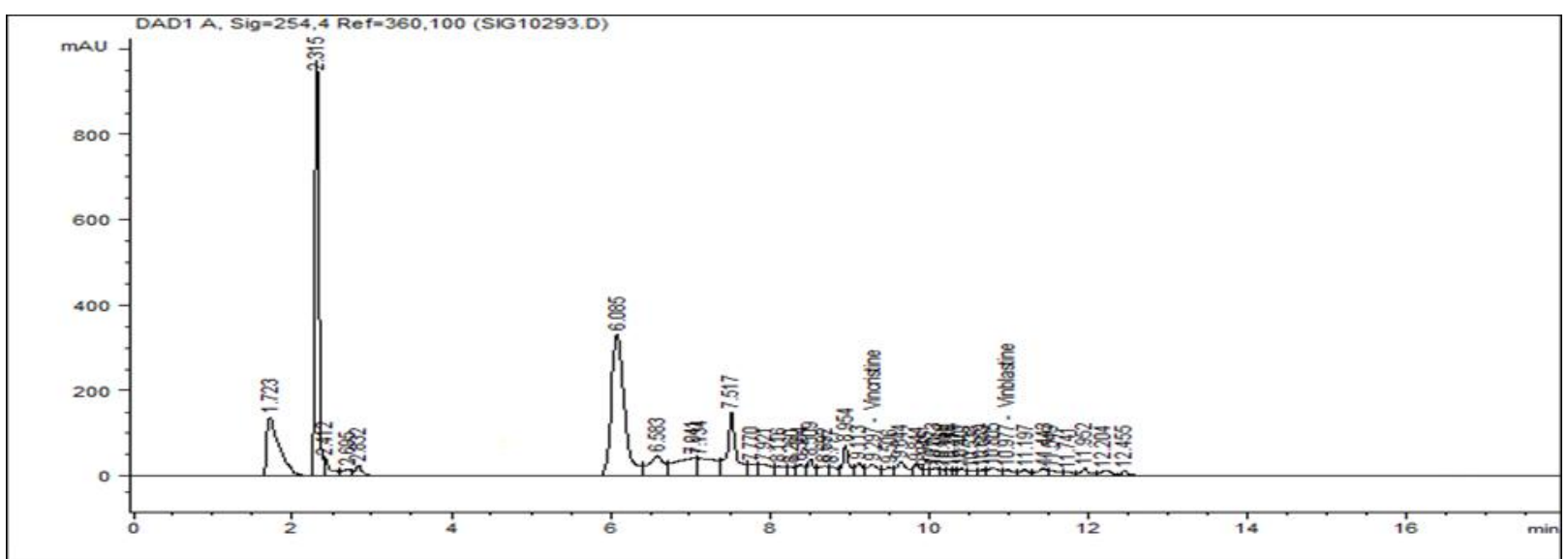

(c)

Figure.5 Comparative analysis of the HPLC-UV254nm secondary metabolite profiles produced

by $P$. concavoradulozum VE89L with and without addition of two epigenetic modifiers in growth medium: (a) - control, (b) - quercetin, (c) - nicotinamide 


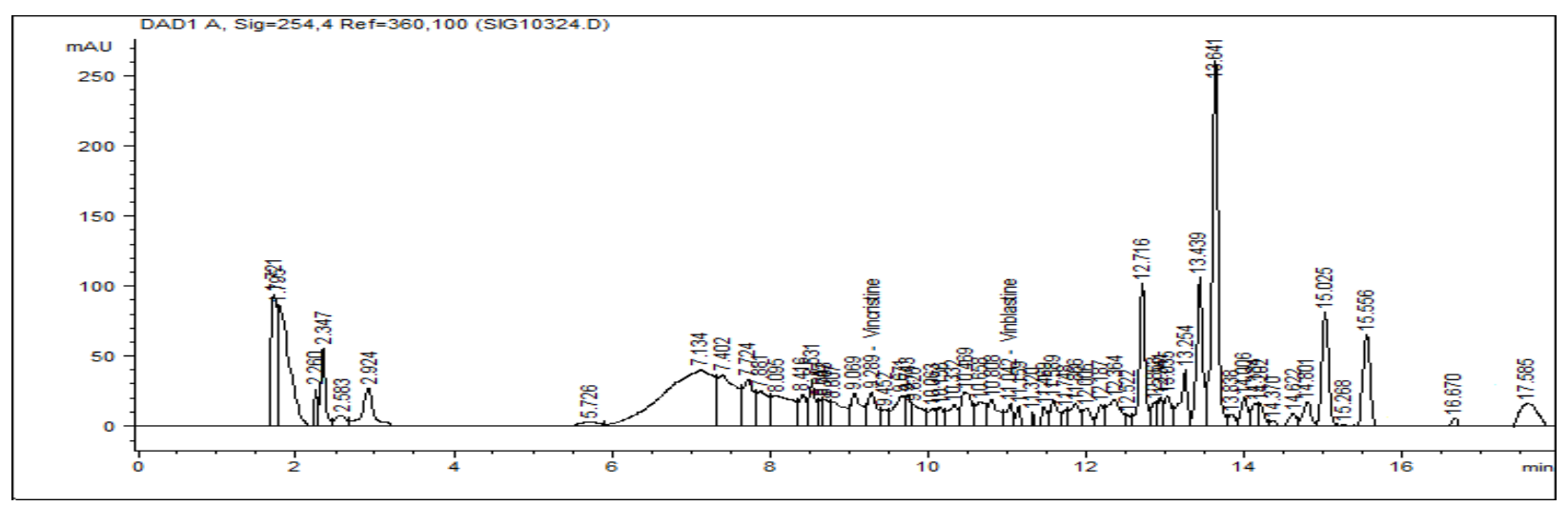

(a)

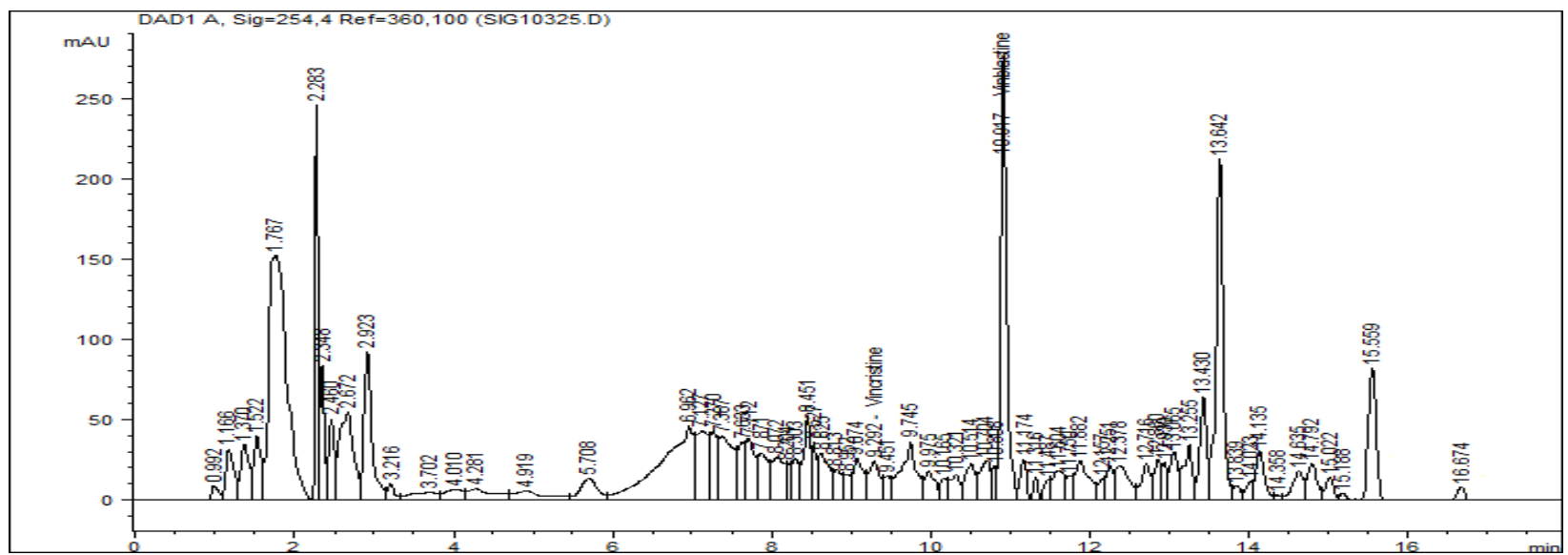

(b)

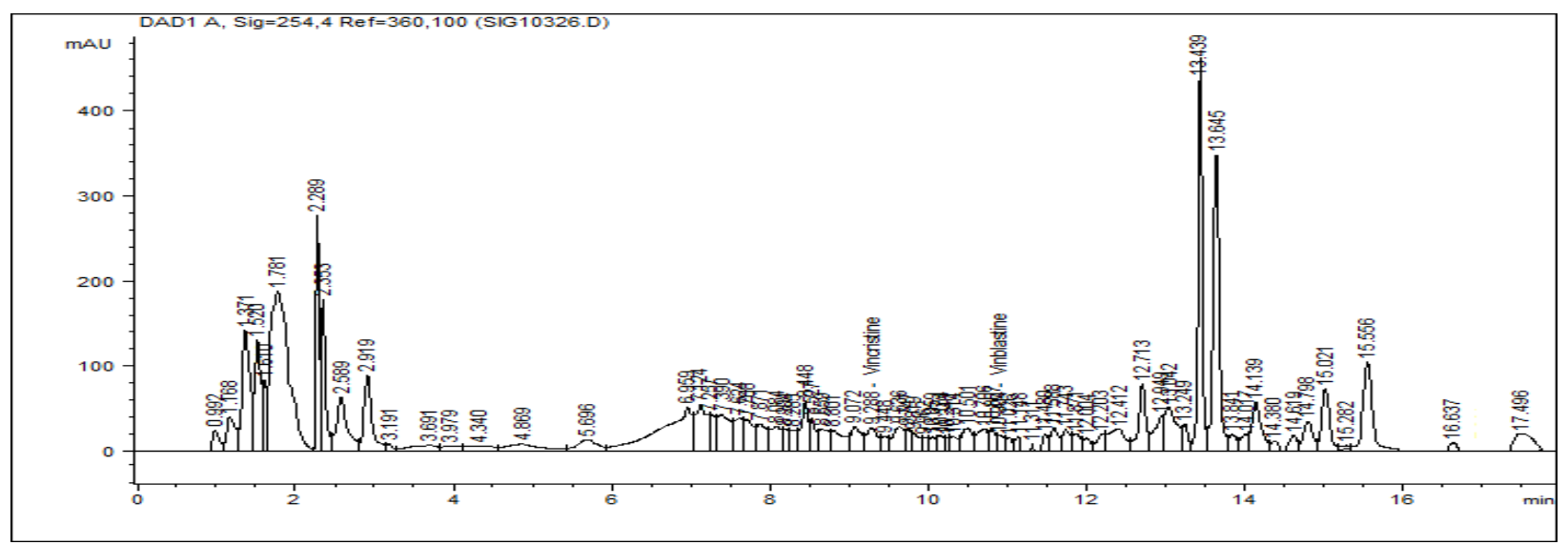

(c)

Figure 6.Comparative analysis of the HPLC-UV254nm secondary metabolite profiles produced by $A$. amstelodami VR177L with and without addition of two epigenetic modifiers in growth medium: (a) - control, (b) - quercetin, (c) - nicotinamide 


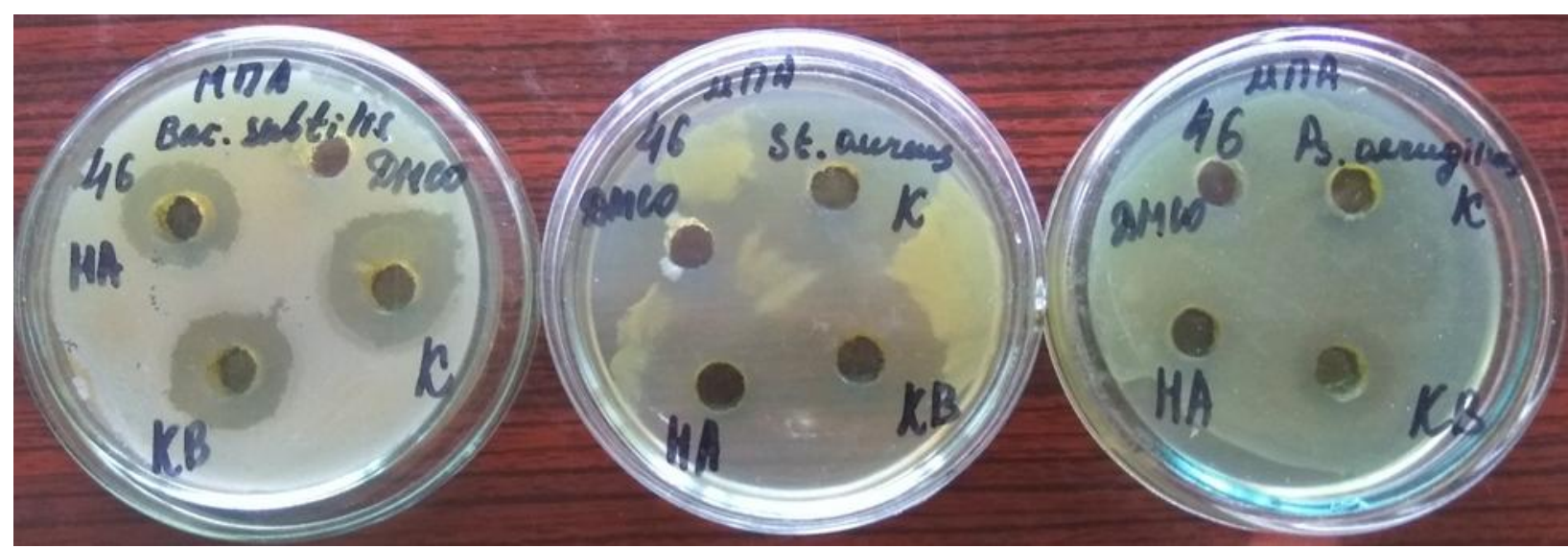

(a)

(b)

(c)

Figure.7 Antibacterial activity of extracts of T. microsporaMO46L cultures grown in addition: KB- quercetin, HA- nicotiamide, K-control media without modificators against (a) - B.subtilis, (b) - S. aureus, (c) - Ps.aerugenosa

Thus, the treatment of endophytic fungi with epigenetic modifiers is an effective tool not only for studying the biotechnological potential of endophytic fungi, but also for increasing their efficiency for production of the target products.

The work was carried out within the framework of the project entitled "Development of conditions for production of bioactive secondary metabolites of endophytic fungi isolated from medicinal plants growing in Uzbekistan", and supported by fundamental and applied research programs of the Academy of Sciences of Uzbekistan.

\section{References}

Abdulmyanova L.I., Fayzieva F.K., Ruzieva D.M., Karimova F.A., Sattarova R.S., Gulyamova T.G. Antimicrobial activity of endophytic fungi from Ferula foetida. 2015. International Journal of Current Microbiology and Applied Science, 4 (11): 154-159.

Abdulmyanova L.I., Teomashko N.N., Terent'eva E.O., Ruzieva D.M., Sattarova R.S., Azimova Sh.S.,
Gulyamova T.G. 2015. Cytotoxic activity of fungal endophytes from Vinca. International Journal of Current Microbiology and Applied Science, 4 (7): 321-329.

Asai T., Morita S., Taniguchi T., Monde K., Oshima Y. 2015. Epigenetic stimulation of polyketide production in Chaetomium cancroideum by an $\mathrm{NAD}(+)$-dependent HDAC inhibitor. Org. Biomol. Chem., 14: 646-651.

Chen, H.J., Awakawa, T., Sun, J.Y., Wakimoto, T., Abe, I. 2013. Epigenetic modifier-induced biosynthesis of novel fusaric acid derivatives in endophytic fungi from Datura stramonium L. Nat. Prod. Bioprospect., 3: 20-23.

Cichewicz, R.H. Epigenome manipulation as a pathway to new natural product scaffolds and their congeners. 2010. Nat. Prod. Rep., 27: 11-22.

Gonzalez-Menendez V., Purez-Bonilla V., Purez-Victoria I., Martin J., Mucoz F., Reyes F., Tormo J.R. , Genilloud O. 2016. Multicomponent Analysis of the Differential Induction of Secondary Metabolite Profiles in Fungal Endophytes. Molecules, 21: 234.

Gross H. 2009. Genomic mining - a concept 
for the discovery of new bioactive natural products. Curr Opin Drug Discov Dev, 2: 207-21.

Hazalin N.A., Ramasamy K., Lim S.M., Wahab I.A., Cole A. Lj, Majeed A.A. 2009. Cytotoxic and antibacterial activities of endophytic fungi isolated from plants at the National Park, Pahang, Malaysia. BMC Complementary and alternative medicine, 9: 46.

Henrikson J.C., Hoover A.R., Joyner P.M., Cichewicz R.H. 2009. A chemical epigenetics approach for engineering the in situ biosynthesis of a cryptic natural product from Aspergillus niger. Org. Biomol. Chem., 7: 435-438.

Hertweck C. 2009. Hidden biosynthetic treasures brought to light. Nat Chem Biol, 5: 450-452.

Lang, G., Blunt, J.W., Cummings, N.J., Cole, A., Lj., Munro, M.H.G. 2005. Hirsutide a cyclic tetrapeptide from a spiderderived entomopathogenic fungus Hirsutella sp. J. Nat. Prod., 68: 1303 1305.

Magotra A., Kumar M., Kushwaha M., Awasthi P., Raina C., Gupta A.P., Shah B.S., Gandhi S.G., Chaubey A. 2017. Epigenetic modifier induced enhancement of fumiquinazoline $\mathrm{C}$ production in Aspergillus fumigatus (GA-L7): an endophytic fungus from Grewia asiatica L. AMB Expr. 7: 43.

Nisa H, Kamili A., Nawchoo I., Shafi S., Shameem N., Bandh S. 2015. Fungal endophytes as prolific source of phytochemicals and other bioactive natural products: A review. Microbial Pathogenesis, 82: 50-59.

Pettit R.K. 2011. Small-molecule elicitation of microbial secondary metabolites. Microbial Biotechnol, 4 (4): 471-478.

Ruzieva D.M., Abdulmyanova L.I., Rasulova G.A., Sattarova R.S. and Gulyamova T.G.. 2017. Screening of Inhibitory Activity against $\alpha$-Amylase of Fungal Endophytes Isolated from Medicinal Plants in Uzbekistan. Int. J. Curr. Microbiol. App. Sci, 6 (4): 2744-2752. Zhang H.W, Song Y.C. and Ren Xiang Tan. 2006. Biology and chemistry of endophytes. Nat. Prod. Rep., 23: 753771.

Sun J., Awakawa T., Noguchi H., Abe I. 2012. Induced production of mycotoxins in an endophytic fungus from the medicinal plant Datura stramonium L. Bioorg Med Chem Lett., 22 (20): 6397-400.

Vervoort, H.C., Draškovicr, M., Crews, P. Histone. 2011. Deacetylase Inhibitors as a Tool to Up-regulate New Fungal Biosynthetic Products - Isolation of EGM-556, a Cyclodepsipeptide, from Microascus sp. Org. Lett., 13: 410-413.

Zhao, J., Shan, T., Mou, Y., Zhou, L., 2011 b. Plant-derived bioactive compounds producedby endophytic fungi. Mini Rev. Med. Chem. 11: 159-168.

\section{How to cite this article:}

Gulyamova, T.G., L.I. Abdulmyanova, D.M. Ruzieva, G.A. Rasulova, U.K. Yusupov and Sattarova, R.S. 2019. Effect of Epigenetic Modifiers on Fermentation Parameters of Endophytic Fungi from Plants Growing in Uzbekistan. Int.J.Curr.Microbiol.App.Sci. 8(03): 851-860. doi: https://doi.org/10.20546/ijcmas.2019.803.102 